\title{
SOLUTION OF SECOND AND THIRD ORDER LINEAR DIFFERENTIAL EQUATIONS BY FRACTIONAL CALCULUS
}

\author{
B. AL-SAQABI AND S.L. KALLA
}

\begin{abstract}
The method of fractional calculus is used to obtain particular solutions of some second and third order linear differential equations. Corresponding homogeneous equations are considered. Some results given recently by Nishimoto and Nishimoto \& kalla follow as particular cases of our theorems.
\end{abstract}

\section{Introduction.}

Fractional calculus deals with derivatives and integrals arbitrary orders, called differintegrals $[1,2,3,4,5]$. Many results about differintegrals of complex order $\nu$ are motivated by the corresponding results of ordinary derivatives and integrals of integer order. There are several definitions of an integral of fractional order, one of the simplest is the Riemann-Liouville operator of fractional integration [2].

$$
\begin{aligned}
R_{x}^{\nu} f=I_{x}^{\nu} & =\frac{1}{\Gamma(\nu)} \int_{0}^{x}(x-t)^{\nu-1} f(t) d t & & \text { for } \operatorname{Re}(\nu)>0 \\
& =\frac{d^{n}}{d x^{n}} R_{x}^{\nu+n} f ; & & \text { for } \operatorname{Re}(\nu)<0
\end{aligned}
$$

Grunwald [1], has defined an another form of the differintegral of order $\nu$

$$
\frac{d^{\nu} f}{[d(x-a)]^{\nu}}=\lim _{N \rightarrow \infty}\left\{\frac{\left[\frac{x-a}{N}\right]^{-\nu}}{\Gamma(-\nu)} \sum_{j=0}^{N-1} \frac{\Gamma(j-\nu)}{\Gamma(j+1)} f\left(x-j\left[\frac{x-a}{N}\right]\right)\right\}
$$

where $\nu$ is arbitrary.

Nishimoto $[3,5]$, defines the differintegral in the following form

$$
f_{\nu}={ }_{c} f_{\nu}(z)=\frac{\Gamma(\nu+1)}{2 \pi i} \cdot \int_{C} \frac{f(\xi)}{(\xi-z)^{\nu+1}} d \xi
$$

Received September 231988

Visiting Professor : Division de Postgrado, Facultad de Ingenieria, Universidad del Zulia, Maracaibo, Venezuela. 
where $f(z)$ is a regular function and it has no branch points inside and on $C(C=$ $\left\{C_{-}, C_{+}\right\}, C_{-}$is an integral curve along the cut joining two points $z$ and $-\infty+i \operatorname{Im}(z)$ and $C_{+}$is an integral curve along cut joining two points $z$ and $\left.\infty+i \operatorname{Im}(z)\right)$

where $\nu \notin Z^{-} ; \nu \in R$ and

$$
f_{-n}=\lim _{\nu \rightarrow-n} f_{\nu} \quad\left(n \in Z^{+}\right)
$$

( $Z^{-}$and $Z^{+}$are the set of negative and positive integers)

$$
\xi \notin z,-\pi \leq \arg (1-z) \leq \pi \quad \text { for } \quad C_{-}
$$

and

$$
0 \leq \arg (\xi-z) \leq 2 \pi \quad \text { for } \quad C_{+}
$$

$f_{\nu}(\nu>0)$ is the fractional derivative of order $\nu$ and $f_{\nu}(\nu<0)$ is the fractional integral of order $\nu$, If $f_{\nu}$ exists, the principal value of $f$ will be considered for many valued functions.

Various authors $[6,7,8,9]$ have defined and studied differintegral operators and their applications.

Recently [9] has studied a generalized $m$-dimensional operators of fractional integration which are defined by means of a convolution type integrals with a suitable Meijer's $G$-function as a kernel, viz. $G_{m, m}^{m, 0}, m \geq 1$.

They can be considered also as compositions of arbitrary numbers $(m \geq 1)$ of commuting Erdelyi-Kober fractional integrals and most of the known differintegral operators becomes as special cases.

Many physical applications of operators are mentioned in [1,4]. Kalla-Ross [10], Kalla-Al-Saqabi [11] have applied fractional calculus in the summation of series.

K. Nishimoto $[12,13,5,14]$ has obtained solutions of several differential equations by using the fractional calculus. Recently Nishimoto and Kalla [15] have applied fractional calculus to solve a third order linear ordinary differential equation.

Fractional differintegration of product $u v[3]$ : Let $u(z)$ and $v(z)$ be analytic and are one valued functions respectively, then $\{u v\}_{\nu}$ is fractional derivative of product $u v$ for $\operatorname{Re}(\nu)>0$, original product $u v$ for $\nu=0$ and fractional integral of product $u v$ for $\operatorname{Re}(\nu)<0$, if $u_{\nu}$ and $v_{\nu}$ exist, where

$$
\begin{gathered}
\{u v\}_{\nu}=\left\{(u v)_{\nu},(v u)_{\nu}\right\} \\
\left\{\begin{array}{l}
(u v)_{\nu}=\sum_{n=0}^{\infty} P(\nu, n) u_{\nu-n} v_{n} \\
(v u)_{\nu}=\sum_{n=0}^{\infty} P(\nu, n) v_{\nu-n} u_{n} \\
\text { and } P(\nu, n)=\frac{\Gamma(\nu+1)}{\Gamma(\nu-n+1) \Gamma(n+1)} \\
z, \nu \in C
\end{array}\right.
\end{gathered}
$$


In this paper, we obtain particular solutions to some second and third order linear ordinary differential equations by invoking the fractional calculus technique. Correponding homogeneous cases are considered. Some known results follow as particular cases of our theorems established here.

2. Solutions of second order linear differential equations.

Theorem 1. If $f_{\nu}(\neq 0)$ exists, then the non-homogeneous second order linear ordinary differential equation

$$
\phi_{2} \cdot\left(z^{2}-\mu z\right)+\phi_{1}(2 \nu z-\mu \nu+\mu)+\phi \nu(\nu-1)=f
$$

has a particular solution of the form

$$
\phi=\left[\left(f_{1-\nu} \frac{z-\mu}{z}\right)_{-1} \frac{1}{(z-\mu)^{2}}\right]_{\nu-2}
$$

where $\phi=\phi(z), f=f(z), z \in C$ and $\mu, \nu$ are constants.

Proof. Putting

$$
\phi=W_{\nu-1}=W_{\nu-1}(z)
$$

yields

$$
\phi_{1}=W_{\nu}
$$

and

$$
\phi_{2}=W_{\nu+1}
$$

The differential equation (7) can be written as follows after using (9), (10), (11), and (6).

$$
\left(w_{1} \cdot z^{2}\right)_{\nu}-\mu\left(w_{2} \cdot z\right)_{\nu-1}=f
$$

By making the differintegral of order $(1-\nu)$ of both sides of $(12)$, we get

$$
w_{2}\left(z^{2}-\mu z\right)+w_{1} \cdot 2 z=f_{1-\nu}
$$

Setting

$$
w_{1}=u(z)
$$

Equation (13) can be written as

$$
\frac{d u}{d z}+\frac{2}{(z-\mu)} u=\frac{f_{1-\nu}}{\left(z^{2}-\mu z\right)}
$$

The first order differential equation (15) has a solution. 


$$
u=\left(f_{1-\nu} \frac{z-\mu}{z}\right)_{-1} \frac{1}{(z-\mu)^{2}}
$$

Hence, from (11), (14) and (16), $\phi(z)$ can be written as given by (8).

Theorem 2. The homogeneous second order linear ordinary differential equation

$$
\phi_{2}\left(z^{2}-\mu z\right)+\phi_{1}(2 \nu z-\mu \nu+\mu)+\phi \nu(\nu-1)=0
$$

has a particular solution of the form

$$
\phi=A\left[(z-\mu)^{-1}\right]_{\nu-1}
$$

where $\phi=\phi(z), z \in C$ and $\mu, \nu$ and $A$ are constants.

Proof. Let

$$
\phi=w_{\nu-1}(z)
$$

This gives

$$
\phi_{1}=w_{\nu}
$$

and

$$
\phi_{2}=w_{\nu+1}
$$

Using (19), (20), (21) and (6) in (17), we get

$$
\left(w_{2}\left(z^{2}-\mu z\right)+w_{1} \cdot 2 z\right)_{\nu-1}=0
$$

or it can be written as

$$
w_{2}\left(z^{2}-\mu z\right)+w_{1} 2 z=0
$$

solution of (23) can be written as

$$
w_{1}=\frac{A_{1}}{(z-\mu)^{2}}
$$

$A_{1}$ is constant, hence, from (19) and (24), the particular solution of (17) is given by (18).

For $\mu=1$, the theorems 1 and 2 reduces to the results given recently by Nishimoto $[5, \mathrm{p} .58]$.

\section{Example of Theorem 1.}

Let $\nu=\frac{1}{2}, \mu=1$ and $f(z)=\frac{1}{z}$

We have from (7) and (8) respectively

$$
\phi_{2}\left(z^{2}-z\right)+\phi_{1}\left(z+\frac{1}{2}\right)-\frac{\phi}{4}=\frac{1}{z}
$$


and

$$
\phi=\left|\left(\left(\frac{1}{z}\right)_{\frac{1}{2}} \frac{z-1}{z}\right)_{-1} \frac{1}{(z-1)^{2}}\right|_{-\frac{3}{2}}
$$

From [3], we can easily show that

$$
\left(\frac{1}{z}\right)_{\frac{1}{2}}=\frac{-i}{2} \sqrt{\pi} z^{-\frac{3}{2}}
$$

and

$$
\left(\frac{-i \sqrt{\pi}}{2} z^{-\frac{5}{2}}(z-1)\right)_{-1}=i \sqrt{\pi}\left(z^{-\frac{1}{2}}-\frac{1}{3} z^{-\frac{3}{2}}\right)
$$

and since

$$
z^{-2}\left(1-\frac{1}{z}\right)^{-2}=\sum_{k=1}^{\infty} k z^{-(k+1)}
$$

Therefore (26) can be written as

$$
\phi=\sqrt{\pi} \sum_{k=1}^{\infty} \frac{\Gamma(k+1)}{\Gamma\left(k+\frac{3}{2}\right)}\left(1-\frac{2 k}{6 k+9}\left(\frac{1}{z}\right)\right) z^{-k}
$$

Theorem 3. If $f_{\nu}(\neq 0)$ exists, then the non-homogeneous second order linear ordinary differential equation

$$
\begin{aligned}
& \phi_{2}\left(z^{2}-\mu z\right)+\phi_{1}(2 \nu z-\mu \nu-\mu)+\phi \nu(\nu-1)=f \\
& z \neq 0, \mu
\end{aligned}
$$

has a particular solution of the form

$$
\phi=\left[\left(f_{-\nu} \frac{1}{(z-\mu)^{2}}\right)_{-1}\left(\frac{z-\mu}{z}\right)\right]_{\nu-1}
$$

where $\phi=\phi(z), z \in C, \mu$ and $\nu$ are constants.

Proof. Setting

$$
\phi=w_{\nu}(z)
$$

yields

$$
\begin{aligned}
& \phi_{1}=w_{\nu+1} \\
& \phi_{2}=w_{\nu+2}
\end{aligned}
$$

Using (31), (32), (33) and (6) in (29), we obtain

$$
\left(w_{2} \cdot z^{2}\right)_{\nu}-\mu\left(w_{2} \cdot z+w_{1}\right)_{\nu}=f
$$


Making the differintegral of both sides of $(34)$ of order $(-\nu)$, we get

$$
w_{2}\left(z^{2}-\mu z\right)-\mu w_{1}=f_{-\nu}
$$

This equation can be written as

$$
\left(w_{1} \frac{z}{z-\mu}\right)_{1}=\frac{f_{-\nu}}{(z-\mu)^{2}}
$$

and

then

$$
w_{1}=\left(\frac{f_{-\nu}}{(z-\nu)^{2}}\right)_{-1}\left(\frac{z-\mu}{z}\right)
$$

$$
w=\left[\left(\frac{f_{-\nu}}{(z-\mu)^{2}}\right)_{-1}\left(\frac{z-\mu}{z}\right)\right]_{-1}
$$

and $\phi$ can be written from (31) as

$$
\phi=\left[\left(\frac{f_{-\nu}}{(z-\mu)^{2}}\right)_{-1}\left(\frac{z-\mu}{z}\right)\right]_{\nu-1} .
$$

Theorem 4. The homogeneous second order linear ordinary differential equation

$$
\begin{aligned}
& \phi_{2}\left(z^{2}-\mu z\right)+\phi_{1}(2 \nu z-\mu \nu-\mu)+\phi \nu(\nu-1)=0 \\
& z \neq 0, \mu
\end{aligned}
$$

where $\phi=\phi(z), z \in C, \mu$ and $\nu$ are constants, has a particular solution

$$
\phi=B(z-\mu / \ln z)_{\nu}
$$

where $B$ is constant

Proof. Letting

$$
\phi=w_{\nu}(z)
$$

yields

$$
\begin{aligned}
& \phi_{1}=w_{\nu+1} \\
& \phi_{2}=w_{\nu+2}
\end{aligned}
$$

We can obtain as in proof of theorem 3 , the following

$$
\left(w_{2} z^{2}\right)_{\nu}-\mu\left(w_{2} z+w_{1}\right)_{\nu}=0
$$

By making the differintegral of both sides of $(44)$ of order $(-\nu)$, we have

$$
w_{2}=\frac{\mu}{z^{2}-\mu z} w_{1}
$$


The solution of $(45)$ is given by

$$
w=B(z-\ln z)
$$

From (41), the solution of (39) can be given in the form of (40).

The results given by Nishimoto [5, p.45] follow from the theorems 3 and 4 , for $\mu=1$.

Theorem 5. If $f_{\nu}(\neq 0)$ exists, then the non-homogeneous second order linear ordinary differential equation

$$
\begin{aligned}
& \phi_{2}(z+b)+\phi_{1}(\nu+1-a b-a z)-\phi a \nu=f \\
& z \neq-b
\end{aligned}
$$

has a particular solution

$$
\phi=\left[\left(f_{-\nu} e^{-a(z+b)}\right)_{-1} \frac{e^{a(z+b)}}{(z+b)}\right]_{\nu-1}
$$

where $\phi=\phi(z), z \in C$ and $\nu, a$ and $b$ are constants.

Proof. Letting

$$
\phi=w_{\nu-1}(z)
$$

yields

$$
\begin{aligned}
& \phi_{1}=w_{\nu} \\
& \phi_{2}=w_{\nu+1}
\end{aligned}
$$

Hence, we can write (47) after using (49), (50), (51) and (6) in the following form

$$
\left(w_{1}(z+b)\right)_{\nu}+(w(1-a b))_{\nu}-a(w z)_{\nu}=f
$$

This equation becomes

$$
w_{1}(z+b)+w((1-a b)-a z)=f_{-\nu}
$$

by making the differintegral of order $(-\nu)$ to both sides of $(52)$.

Integrating (53), we can easily get

$$
w=\left(f_{-\nu} e^{-a(z+b)}\right)_{-1} \frac{e^{a(z+b)}}{(z+b)}
$$

Finally, we can obtain the particular solution of (47) in the form given by (48) by use of (49). 
Theorem 6. The homogeneous second order linear ordinary differential equation

$$
\phi_{2}(z+b)+\phi_{1}(\nu+1-a b-a z)-\phi a_{\iota}^{\prime}=0
$$

has a particular solution

$$
\phi=\left(\frac{K e^{a z}}{z+b}\right)_{\nu-1}
$$

where $\phi=\phi(z), z \in C, \nu, a, b$ and $K$ are constants.

Proof. By following the same method as in obtaining equation (53); we have

$$
w_{1}(z+b)+w((1-a b)-a z)=0
$$

Integrating (57), we obtain

$$
w=K \frac{e^{a z}}{(z+b)},
$$

Hence, from (49), the particular solution of (55) is in the form of (56).

3. Solution of a third order linear ordinary differential equation.

Theorem 7. If $f_{\alpha}(\neq 0)$ exists, then non-homogeneous third order linear ordinary differential equation

$$
\begin{aligned}
\phi_{3}\left(z^{3}-\delta^{2} z\right) & +\phi_{2}\left\{(3 \alpha+\beta+\gamma) z^{2}+\beta \gamma z-\alpha \delta^{2}\right\} \\
& +\phi_{1}\{\alpha(3 \alpha+2 \beta+2 \gamma-3) z+\alpha \beta \gamma\} \\
& +\phi \alpha(\alpha-1)(\alpha+\beta+\gamma-2)=f \\
(z \neq 0, \pm \delta) &
\end{aligned}
$$

has a particular solution

$$
\phi=\left(\left(f_{-\alpha} \frac{(z-\delta)^{A}(z+\delta)^{B}}{z^{3}-\delta^{2} z}\right)_{-1} \frac{1}{(z-\delta)^{A}(z+\delta)^{B}}\right)_{\alpha-2}
$$

where

$$
\phi=\phi(z), f=f(z), z \in C, \alpha, \beta, \gamma \text { and } \delta^{2} \text { are constants and }
$$

$$
A=\frac{1}{2}\left(\beta+\gamma+\frac{\beta \gamma}{\delta}\right) ; \quad B=\frac{1}{2}\left(\beta+\gamma-\frac{\beta \gamma}{\delta}\right) \text {. }
$$

Proof. Putting

$$
\phi=w_{\alpha}=w_{\alpha}(z)
$$

yields

$$
\begin{aligned}
& \phi_{1}=w_{\alpha+1} \\
& \phi_{2}=w_{\alpha+2} \\
& \phi_{3}=w_{\alpha+3}
\end{aligned}
$$


By substituting (61)-(64) and (6) in (59), we obtain

$$
\left(w_{3} z^{3}\right)_{\alpha}-\delta^{2}\left(w_{3} z\right)_{\alpha}+(\beta+\gamma)\left(w_{2} z^{2}\right)_{\alpha}+\beta \gamma\left(w_{2} z\right)_{\alpha}=f
$$

Therefore, we can write (65) as follows

$$
w_{3}+w_{2} \frac{(\beta+\gamma) z+\beta \gamma}{z^{2}-2}=\frac{f_{-\alpha}}{z^{3}-\delta^{2} z}
$$

Let

$$
w_{2}=u(z)
$$

we have

$$
u_{1}+u \cdot \frac{(\beta+\gamma) z+\beta \gamma}{z^{2}-\delta^{2}}=\frac{f_{-\alpha}}{z^{3}-\delta^{2} z}
$$

A particular solution of (68) is given in the form (60).

For $\delta=1$, theorem 7 , gives the non-homogeneous third order linear differential equation considered by Nishimoto and Kalla [15].

Theorem 8. Homogeneous third order linear ordinary differential equation of the form

$$
\begin{aligned}
\phi_{3}\left(z^{3}-\delta^{2} z\right) & +\phi_{2}\left\{(3 \alpha+\beta+\gamma) z^{2}+\beta \gamma z-\alpha \delta^{2}\right\} \\
& +\phi_{1}\{\alpha(3 \alpha+2 \beta+2 \gamma-3) z+\alpha \beta \gamma\} \\
& +\phi \alpha(\alpha-1)(\alpha+\beta+\gamma-2)=0 \\
(z \neq 0, \pm \delta) &
\end{aligned}
$$

has a solution of the form

$$
\phi=\left(\frac{1}{(z-\delta)^{A}(z+\delta)^{B}}\right)_{\alpha-2}
$$

where $\phi=\phi(z)$ and $z \in C$.

$A$ and $B$ are defined in Theorem 7 .

Proof. Following the same steps in proof theorem 7 , we obtain

$$
w_{3}+w_{2} \frac{(\beta+\gamma) z+\beta \gamma}{z^{2}-\delta^{2}}=0
$$

Therefore, we have

$$
u_{1}+u \frac{(\beta+\gamma) z+\beta \gamma}{z^{2}-\delta^{2}}=0
$$

where

$$
w_{2}=u(z)=u
$$


And the solution of (72) is given by

$$
\phi=w_{\alpha}=u_{\alpha-2}=\left(\frac{1}{(3-\delta)^{A}(z+\delta)^{B}}\right)_{\alpha-2} .
$$

\section{Acknowledgement}

This work is supported by Kuwait Foundation for Advancement of Science through research project no. 88-09-02.

\section{References}

[1] K.B.Oldham and J. Spanier. The Fractional Calculus, Academic Press, New York, 1974.

[2] B. Ross (Editor). Fractional Calculus, Research Notes in Math \#457, Springer-Verlag, 1975.

[3] K. Nishimoto, Fractional Calculus. Descartes Press, Koriyama, Japan, 1984.

[4] A.C. McBride and G.F. Roach (Editors), Fractional Calculus, Pitman, London, 1985.

[5] K. Nishimoto. Fractional Calculus, Vol.II, Descartes Press, Koriyama, Japan 1987.

[6] M.Saigo. A remark on integral operators involving the Gauss' hypergeometric functions. Math. Rep. College General Ed. Kyushu Univ. 11(1978), 135-143.

[7] S.L. Kalla. Operators of fractional integration, In "Analytic Functions": Kozubnik, 1979, Lecture Notes in Mathematics \# 798, Springer-Verlag.

[8] I.H. Dimovski and V.S. kiryakova. Transmutations, Convolutions and fractional powers of BesselType operators via Meijer's G-function. Proc. Complex Anal. and appl., Varna 1985, 45-66.

[9] V.S. Kiryakova. Generalized Operators of Fractional Integration \& Differentiation and Applications. Doctoral dissertationa, Inst.Math.,Bulgarin Acad. Sci. Sofia, 1987.

[10] S.L. Kalla and B. Ross. The development of functional relations by means of fractional operators. In "Fractional Calculus", Pitman London, 1985.

[11] S.L. Kalla and B. Al-Saqabi. A functional relation involving $\psi$-function. Rev. Tec., Univ. Zulia, 8(ii), 1985, p.31-35.

[12] K. Nishimoto. Application of fractional calculus to the solutions of linear second order differential equations of Fuchs type, In "Fractional Calculus", Pitman, London, 1985, p.140-153.

[13] K. Nishimoto. An application of fractional calculus to the nonhomogeneous Gauss' equation. Jour. Coll. Eng. Nihon Univ. B-28 (1987), p.1-8.

[14] K. Nishimoto. Fractional calculous and its applications to the non-homogeneous Gauss' equations., Rev. Tec., Univ. Zulia, Vol.10, no.1, Edicion Especial, 1987.

[15] K. Nishimoto and S.L. Kalla. Use of fractional calculus to solve certain linear differential equations. Jour. Coll. Engg., Nihon univ.

Department of Mathematics, Kuwait University, PO. Box 5969 Safat KUWAIT. 